Artigo Original

Original Article

Rosângela Ghiringhelli ${ }^{1}$

Cristina Freitas Ganança ${ }^{1}$

Descritores

Doenças vestibulares

Postura

Tontura

Equilíbrio postural Avaliação

Keywords

Vestibular diseases

Posture

Dizziness

Postural balance

Evaluation

Endereço para correspondência:

Cristina Freitas Ganança

R. Botucatu, 802, São Paulo (SP), Brasil, CEP: 04023-900.

E-mail: crisfreitasg@hotmail.com

Recebido em: 23/9/2009

Aceito em: 23/3/2010

\section{Posturografia com estímulos de realidade virtual em adultos jovens sem alterações do equilíbrio corporal}

\author{
Posturography with virtual reality stimulations in normal \\ young adults with no balance complaints
}

\section{RESUMO}

Objetivo: Analisar os achados à posturografia com estímulos de realidade virtual realizada em adultos jovens, hígidos e sem queixas otoneurológicas, de acordo com parâmetros de centro de pressão, limite de estabilidade e velocidade da oscilação para diferentes estímulos visuais e em relação aos gêneros masculino e feminino. Métodos: Participaram 50 indivíduos hígidos de ambos os gêneros (50\% gênero feminino e 50\% masculino), com idades entre 18 e 25 anos (média de idade 21,30 anos), sem queixas relacionadas ao equilíbrio corporal e avaliação vestibular com vectonistagmografia digital dentro dos padrões de normalidade. A posturografia foi composta por 11 estímulos visuais e determinou a área do limite de estabilidade (LOS), área de elipse, e velocidade de oscilação em dez condições sensoriais. Os resultados foram calculados para a faixa etária estudada e analisados quanto aos valores para cada estímulo, visando à obtenção de limites de normalidade dos parâmetros. Resultados: Os valores médios obtidos na posturografia, quanto ao limite de estabilidade, à área da elipse e à velocidade de oscilação para os estímulos com interação visuo-vestibular apresentaram diferenças entre os gêneros, sendo que em todos os casos as mulheres obtiveram valores inferiores aos dos homens. Conclusão: Os achados à posturografia com estímulos de realidade virtual em adultos jovens hígidos evidenciam que os parâmetros centro de pressão, limite de estabilidade e velocidade da oscilação apresentam diferenças entre os gêneros, e portanto, devem ser considerados separadamente.

\begin{abstract}
Purpose: To analyze the findings of posturography with virtual reality stimuli carried out in young healthy adults with no otoneurological complaints, based on the parameters center of pressure, limit of stability and sway speed for different visual stimuli, and regarding differences between female and male genders. Methods: Participants were 50 healthy individuals of both genders (50\% female and 50\% male) with ages ranging from 18 to 25 years (mean age 21.30 years), with no complaints regarding body balance, and with vestibular outcomes assessed through digital vectonistagmography within normal limits. Posturography was composed of 11 visual stimuli and determined the limit of stability area (LOS), the ellipse area, and the sway speed in ten sensorial conditions. Results were calculated for the studied age range and analyzed according to the values for each stimulus, with the aim to obtain normality parameters. Results: The average values obtained in posturography regarding limit of stability, ellipse area and sway speed for stimuli with viso-vestibular interaction presented significant differences between genders, and, in all cases, women obtained lower values than men. Conclusion: The findings of posturography with virtual reality stimuli in healthy young adults evidence that the parameters center of pressure, limit of stability and sway speed present differences between genders and, therefore, must be considered separately.
\end{abstract}

Trabalho realizado nos Ambulatórios de Equilibriometria e de Reabilitação Vestibular da Disciplina de Otoneurologia, Departamento de Otorrinolaringologia, Universidade Federal de São Paulo - UNIFESP - São Paulo (SP), Brasil.

(1) Curso de Graduação em Fonoaudiologia, Universidade Federal de São Paulo - UNIFESP - São Paulo (SP), Brasil. 


\section{INTRODUÇÃO}

O equilíbrio corporal é uma complexa interação entre os sistemas sensorial e motor permitindo que o corpo se mantenha parado de modo estável ou em movimento de maneira harmônica e precisa. Quando ocorre uma alteração em um de seus componentes (proprioceptivo - percepção da postura e da movimentação do corpo; vestibular - posição e movimento da cabeça, e visual - relações espaciais), surgem alterações que caracterizam o desequilíbrio e podem afetar a qualidade de vida do indivíduo ${ }^{(1)}$.

A posturografia permite avaliar e treinar pacientes com desordens de equilíbrio, tonturas ou instabilidade. Ela complementa os testes convencionais da avaliação otoneurológica fornecendo informações não somente do sistema vestibular, mas dos demais sistemas que contribuem para a manutenção do equilíbrio( ${ }^{(2)}$.

Define-se como posturografia qualquer estudo ou técnica que avalie a oscilação do corpo ou de uma variável associada a essa oscilação $0^{(3)}$. A medida posturográfica comumente utilizada na avaliação do controle postural é o centro de pressão $(\mathrm{CoP})$, que é o ponto de aplicação da resultante das forças verticais agindo sobre a superfície de suporte e representa um resultado coletivo do sistema de controle postural e da força da gravidade. O CoP é medido por uma plataforma de força, que consiste de uma placa sob a qual sensores de força estão dispostos de tal forma que medem os componentes da força e do momento de força, nas direções ântero-posterior, médio-lateral e vertical, agindo sobre a plataforma ${ }^{(4)}$.

Diversos autores utilizaram a posturografia dinâmica computadorizada na avaliação de diferentes doenças ou sintomas vestibulares relacionados ao equilíbrio corporal e reforçaram sua importância na avaliação otoneurológica ${ }^{(2,5-9)}$. Porém, na Fonoaudiologia ainda são escassas as pesquisas nessa área ${ }^{(10)}$.

A posturografia com estímulos de realidade virtual contribui na identificação das manifestações relacionadas ao desequilíbrio, por meio de estímulos sensoriais projetados em óculos de realidade virtual que simulam situações da vida real. Pode contribuir também para a reabilitação vestibular, provocando situações que causam tontura ou vertigem, o que levaria à adaptação do sistema vestibular ${ }^{(11)}$.

A realidade virtual permite recriar ao máximo a sensação de interação do indivíduo com o computador. Possibilita a imersão em um mundo ilusório, onde a percepção do ambiente é modificada por um estímulo sensorial artificial, o qual pode provocar um conflito vestíbulo-ocular e a mudança do ganho deste mesmo reflexo ${ }^{(12)}$.

A utilização da plataforma de posturografia com estímulos de realidade virtual é um novo método de avaliação. O número de publicações sobre o tema é escasso e não há padrões de referências. Por isso, torna-se necessária a determinação de tais padrões, em diferentes faixas etárias, que servirá de base para futuras pesquisas e avaliações com diferentes grupos de pacientes.

Assim, este estudo busca analisar os achados à posturografia com estímulos de realidade virtual realizada em adultos jovens, hígidos e sem queixas otoneurológicas, de acordo com os parâ- metros de centro de pressão, limite de estabilidade e velocidade da oscilação para diferentes estímulos visuais.

\section{MÉTODOS}

Esta pesquisa foi realizada entre os anos de 2008 e 2009 nos Ambulatórios de Equilibriometria e de Reabilitação Vestibular da Disciplina de Otoneurologia do Departamento de Otorrinolaringologia da Universidade Federal de São Paulo (UNIFESP). Trata-se de estudo clínico prospectivo aprovado pelo Comitê de Ética em Pesquisa da UNIFESP sob o número 1398/07.

Foram incluídos 50 sujeitos, com idades entre 18 e 25 anos, sem queixas relacionadas ao equilíbrio corporal e com capacidade de se manter na posição bípede sem auxilio, sendo 25 indivíduos do gênero masculino e 25 do gênero feminino. $\mathrm{O}$ grupo feminino apresentou média etária de 22 anos $(\mathrm{DP}=1,96)$ e o grupo masculino apresentou média etária de 20,6 anos $(\mathrm{DP}=1,98)$. Não foi verificada diferença significativa entre os grupos em relação à faixa etária. Todos os participantes deveriam apresentar os resultados da Avaliação Otoneurológica (anamnese, audiometria tonal e vocal, imitanciometria e exame vestibular com a vectonistagmografia computadorizada) dentro dos padrões de normalidade.

Foram excluídos do estudo indivíduos que utilizassem qualquer tipo de órtese ou apresentasse sinais ou sintomas atuais ou pregressos de qualquer doença relacionada à disfunção do sistema vestibular.

Todos os participantes realizaram a posturografia computadorizada com estímulos de realidade virtual, baseada em 11 estímulos visuais, controlados pelo computador. Os estímulos foram apresentados em lentes de óculos de realidade virtual acoplado à cabeça do sujeito.

A posturografia foi realizada por meio de uma plataforma de equilíbrio (BRUTM) que converte a pressão aplicada sobre uma superfície plana em sinais elétricos para determinar a área do centro de pressão $(\mathrm{CoP})$ ou centro de massa, o limite de estabilidade do paciente (LOS) e a velocidade de oscilação ${ }^{(10)}$.

A plataforma de pressão possui uma área de $1600 \mathrm{~cm}^{2}$ (40 $\mathrm{cm}$ x $40 \mathrm{~cm}$ ), incluindo coordenadas verticais e horizontais. Além disso, dispõe de uma linha horizontal de $8 \mathrm{~cm}$ (linha intermaleolar) para o posicionamento dos pés do indivíduo e uma linha vertical de $12 \mathrm{~cm}$ para interceptar o ponto médio da linha intermaleolar.

Para a realização da posturografia, o participante deveria estar sobre uma plataforma de força, descalço, em apoio bipodal e com os braços relaxados ao longo do corpo. Foi permitida a utilização de lentes corretivas visuais quando necessário. Durante o procedimento, todos foram orientados a manter postura ereta e estável, permanecendo imóvel.

Inicialmente foi realizada a marcação do limite de estabilidade. O indivíduo foi instruído a deslocar o corpo em sentido ântero-posterior e lateral utilizando a estratégia de tornozelo, sem movimentar os pés ou empregar estratégias de tronco. A movimentação deveria acontecer lentamente até que o participante alcançasse seu limite de estabilidade corporal, respeitando a seguinte sequência: para frente e retornar à posição inicial; para a direita e retornar à posição inicial; para a esquerda e 
retornar à posição inicial; para trás e retornar à posição inicial. Os sujeitos foram orientados a realizar a sequência completa de movimentos por duas vezes, não sendo necessário completar os 60 segundos determinados como tempo máximo para este procedimento. Caso o sujeito movesse os pés ou o tronco, o procedimento era reiniciado.

Os estímulos sensoriais apresentados durante o exame foram visuais (olhos abertos, olhos fechados, estímulos visuais específicos - objetos móveis apresentados em óculos de realidade virtual que recriaram versões estilizadas de situações da vida real), vestibulares (objetos apresentados nos óculos de realidade virtual moviam-se de acordo com os movimentos da cabeça feitos pelo paciente) ou somatossensoriais (uso de colchonete) para mudar a superfície de apoio.

O exame de posturografia foi composto por 11 estímulos visuais (que ocorreram durante $60 \mathrm{~s}$, com intervalo de $2 \mathrm{~s}$ entre eles) em que o participante deveria ficar na posição ortostática. Os estímulos foram: LOS (área do limite de estabilidade) olhos abertos; de pé sobre piso firme - olhos abertos e depois fechados; de pé sobre um colchonete - olhos fechados; sacádico (piso firme) - diversas direções; barras optocinéticas (piso firme) - direções horizontal (da esquerda à direita e da direita à esquerda) e vertical (de cima para baixo e de baixo para cima); interação vestíbulo-visual (piso firme) - trem circular - direções horizontal (da esquerda para a direita) e vertical (de baixo para cima) (Quadro 1).

Uma almofada de espuma de densidade média foi empregada na avaliação com colchonete. Os óculos de realidade virtual foram utilizados nas avaliações seguintes ao uso do colchonete.

Relatórios foram gerados pelo programa contendo informações sobre a área do Limite de Estabilidade, a área de elipse de confiança 95\% e a velocidade de oscilação nas dez condições sensoriais. A área de elipse de confiança 95\% é definida como a área de distribuição de $95 \%$ das amostras do centro de pressão. A velocidade de oscilação média é determinada pela distância total dividida pelo tempo de 60 segundos da prova ${ }^{(11)}$.

Os resultados da posturografia foram coletados a partir da informação do CoP por meio da plataforma de equilíbrio, para cada estímulo, visando estabelecer limites de normalidade dos parâmetros da posturografia (área do LOS, área da elipse e velocidade de oscilação em cada estímulo). Cada parâmetro foi analisado separadamente e em conjunto, a fim de se observar o desempenho de adultos jovens hígidos nesta prova.

Todos os resultados foram submetidos à análise estatística descritiva para caracterização da amostra. O teste de MannWhitney foi utilizado para comparar os resultados entre gênero e as variáveis de limite da área do LOS, área de elipse e velocidade de oscilação, nas condições do BRUTM. O nível de significância adotado foi de $5 \%(\mathrm{p}=0,05)$. Todos os intervalos de confiança construídos ao longo da pesquisa foram definidos com $95 \%$ de confiança estatística.

\section{RESULTADOS}

Em relação aos valores relacionados à área do Limite de Estabilidade (LOS) e à velocidade de oscilação, verificou-se que as variações entre os gêneros foram pequenas (coeficiente de
Quadro 1. Protocolo para realização da posturografia

1. LOS, olhos abertos:
Demora $=2$
Duração $=180 \mathrm{~s}$

2. De pé, sobre piso firme, com olhos abertos:

Demora $=2$

Duração $=60 \mathrm{~s}$

3. De pé sobre piso firme, com olhos fechados:

Demora $=2$

Duração $=60 \mathrm{~s}$

4. De pé sobre um colchonete, com olhos abertos:

Demora $=2$

Duração $=60 \mathrm{~s}$

5. De pé sobre piso firme, sacádico:

Demora $=2$

Duração $=60 \mathrm{~s}$

Altura $=7$

Frequência $=1 \mathrm{~Hz}$

Direção = aleatória

6. De pé sobre piso firme, barras optocinéticas - direção:

horizontal

Demora $=2$

Duração $=60 \mathrm{~s}$

Altura $=7$

Velocidade $=60$

Direção $=$ da esquerda à direita

7. De pé sobre piso firme, barras optocinéticas - direção:

horizontal
Demora $=2$
Duração $=60 \mathrm{~s}$
Altura $=7$
Velocidade $=60$
Direção = da direita à esquerda

8. De pé sobre piso firme, barras optocinéticas - direção: vertical Demora $=2$

Duração $=60 \mathrm{~s}$

Altura $=7$

Velocidade $=60$

Direção = de cima para baixo

9. De pé sobre piso firme, barras optocinéticas - direção: vertical

Demora $=2$

Duração $=60 \mathrm{~s}$

Altura $=7$

Velocidade $=60$

Direção = de baixo para cima

10. De pé sobre piso firme, optocinético vestibular - trem circular

- direção: horizontal

Demora $=2$

Duração $=60 \mathrm{~s}$

Altura $=7$

Velocidade $=60$

Direção = da esquerda para a direita

11. De pé sobre piso firme, optocinético vestibular - trem circular - direção: vertical

Demora $=2$

Duração $=60 \mathrm{~s}$

Altura $=7$

Velocidade $=60$

Direção $=$ de baixo para cima

Fonte: BRU ${ }^{T M}$, Medicaa $\AA$ 
variação próximo a 50\%). Já para os valores da Área de Elipse, estas variações foram consideradas altas, caracterizando a não homogeneidade dos dados. Os valores obtidos foram maiores no gênero masculino, com diferença em relação ao gênero feminino (Tabela 1).

Foram obtidos os valores descritivos e a análise comparativa dos valores do LOS, da área de elipse $\left(\mathrm{cm}^{2}\right)$ e da velocidade de oscilação $(\mathrm{cm} / \mathrm{s})$ nos grupos dos gêneros masculino e feminino (Tabela 2). Houve diferença entre os gêneros para o LOS, sendo que as mulheres apresentaram resultados inferiores aos dos homens. Verificou-se também que houve diferença entre os gêneros quanto à área de elipse, para as seguintes condições do BRUTM: superfície firme com interação visuo-vestibular nas direções horizontal e vertical. Em todos os casos de diferença, as mulheres obtiveram valores inferiores aos dos homens. Para a velocidade de oscilação, em superfície firme com interação visuo-vestibular nas direções horizontal e vertical, observou-se diferenças entre os gêneros, sendo os valores do gênero feminino também inferiores aos do gênero masculino.

\section{DISCUSSÃO}

A posturografia pode ser útil para detecção de disfunções vestibulares, Estudos mostram que as informações sobre as alterações dos valores da velocidade de oscilação e da área de elipse na posturografia podem ser relevantes para a programação e o acompanhamento do tratamento dos distúrbios de equilíbrio corporal ${ }^{(16,19-21)}$.

No presente estudo, foi utilizada a avaliação por posturografia estática. Porém, encontra-se na literatura maior número de estudos que utilizaram a posturografia dinâmica. Tal fato prejudicou a comparação entre dos resultados.

Um estudo $^{(22)}$ utilizando a posturografia estática quantificou e analisou as oscilações posturais de jovens, adultos e idosos, cuja tarefa era permanecer parado em apoio bipodal durante 60 $\mathrm{s}$, inicialmente com olhos abertos e depois com olhos fechados. Os autores não observaram diferenças quanto às oscilações entre os grupos. No entanto, houve diferença entre as oscilações visuais, sendo maiores os valores obtidos nas tarefas com olhos fechados.

Este estudo buscou apresentar valores de referência para o uso da posturografia com estímulos de realidade virtual em adultos jovens hígidos em relação aos gêneros feminino e masculino, frente à necessidade de se criar padrões de normalidade em diferentes faixas etárias, para servir de base para futuras pesquisas com diferentes grupos de pacientes.

Tabela 1. Valores descritivos e análise comparativa da área do Limite de Estabilidade - LOS $\left(\mathrm{cm}^{2}\right)$, área da elipse $\left(\mathrm{cm}^{2}\right)$ e velocidade de oscilação $(\mathrm{cm} / \mathrm{s})$ das condições do Balance Rehabilitation Unit (BRU ${ }^{\mathrm{TM}}$ ) de indivíduos do gênero masculino e feminino

\begin{tabular}{|c|c|c|c|c|c|c|c|c|c|c|}
\hline Descritiva & & Média & Mediana & DP & CV & Q1 & Q3 & Min & Max & IC \\
\hline Área de estabilidade & & 236,2 & 233,0 & 62,4 & $26,4 \%$ & 187,0 & 285,8 & 135,0 & 375,0 & 17,3 \\
\hline \multirow{8}{*}{ Área de elipse $\left(\mathrm{cm}^{2}\right)$} & SFOA & 2,18 & 1,87 & 1,30 & $59,7 \%$ & 1,48 & 2,64 & 0,55 & 7,37 & 0,36 \\
\hline & SEOF & 8,83 & 7,40 & 5,52 & $62,6 \%$ & 5,35 & 10,82 & 3,58 & 37,91 & 1,53 \\
\hline & SFS & 1,77 & 1,25 & 1,46 & $82,5 \%$ & 0,82 & 2,30 & 0,25 & 7,48 & 0,41 \\
\hline & SFOD & 2,09 & 1,60 & 1,83 & $87,5 \%$ & 1,11 & 2,14 & 0,30 & 10,90 & 0,51 \\
\hline & SFOB & 1,78 & 1,55 & 1,28 & $72,2 \%$ & 0,81 & 2,41 & 0,47 & 6,49 & 0,36 \\
\hline & SFOC & 1,96 & 1,59 & 1,45 & $74,0 \%$ & 0,89 & 2,82 & 0,46 & 7,03 & 0,40 \\
\hline & SFIVVDH & 3,46 & 2,43 & 3,51 & $102 \%$ & 1,86 & 3,97 & 0,47 & 19,48 & 0,97 \\
\hline & SFIVVDV & 3,93 & 2,71 & 3,28 & $83,3 \%$ & 1,59 & 5,57 & 0,69 & 14,27 & 0,91 \\
\hline \multirow{7}{*}{ Velocidade de oscilação $(\mathrm{cm} / \mathrm{s})$} & SFOA & 0,75 & 0,66 & 0,43 & $57,8 \%$ & 0,58 & 0,78 & 0,42 & 3,50 & 0,12 \\
\hline & SFOD & 0,81 & 0,76 & 0,26 & $32,1 \%$ & 0,64 & 0,92 & 0,44 & 1,65 & 0,07 \\
\hline & SFOE & 0,82 & 0,75 & 0,28 & $34,3 \%$ & 0,61 & 0,94 & 0,42 & 1,73 & 0,08 \\
\hline & SFOB & 0,84 & 0,82 & 0,27 & $31,9 \%$ & 0,63 & 0,98 & 0,45 & 1,64 & 0,07 \\
\hline & SFOC & 0,87 & 0,78 & 0,35 & $39,6 \%$ & 0,65 & 0,95 & 0,43 & 1,92 & 0,10 \\
\hline & SFIVVDH & 1,24 & 1,11 & 0,53 & $42,6 \%$ & 0,93 & 1,34 & 0,61 & 3,00 & 0,15 \\
\hline & SFIVVDV & 1,52 & 1,39 & 0,58 & $38,1 \%$ & 1,13 & 1,83 & 0,61 & 3,15 & 0,16 \\
\hline
\end{tabular}

\footnotetext{
* Teste de Mann-Whitney $(p \leq 0,05)$
}

Legenda: DP = desvio-padrão; CV = coeficiente de variação; Q1 = distribuição até $25 \%$ da amostra; Q3 = distribuição até $75 \%$ da amostra; Min = valor mínimo da amostra; Max = valor máximo da amostra; IC = ilntervalo de confiança; SFOA = superfície firme de olhos abertos; SFOF = superfície firme de olhos fechados; SEOF = superfície espuma de olhos fechados; SFS = superfície firme movimento sacádico; SFOD = superfície firme barras optocinéticas na direção horizontal da esquerda à direita; SFOE = superfície firme barras optocinéticas na direção horizontal da direita à esquerda; SFOB = superfície firme barras optocinéticas na direção vertical de cima para baixo; SFOC = superfície firme barras optocinéticas na direção vertical de baixo para cima; SFIVVDH = superfície firme interação vestíbulo-visual na direção horizontal da esquerda à direita; SFIVVDV = superfície firme interação vestíbulo-visual na direção vertical de baixo para cima 
Tabela 2. Valores descritivos e análise comparativa da área do Limite de Estabilidade - LOS $\left(\mathrm{cm}^{2}\right)$, da área de elipse $\left(\mathrm{cm}^{2}\right)$ e da velocidade de oscilação (cm/s) das condições do Balance Rehabilitation Unit (BRU $\left.{ }^{\mathrm{TM}}\right)$ de indivíduos do gênero masculino e feminino

\begin{tabular}{|c|c|c|c|c|c|c|}
\hline \multicolumn{2}{|l|}{ Descritiva } & \multirow{3}{*}{$\begin{array}{c}\text { Gênero } \\
\mathrm{F} \\
\mathrm{M}\end{array}$} & \multirow{3}{*}{$\begin{array}{c}\text { Média } \\
207,1 \\
265,2\end{array}$} & \multirow{3}{*}{$\begin{array}{c}\text { Mediana } \\
200,0 \\
272,0\end{array}$} & \multirow{3}{*}{$\begin{array}{l}\text { DP } \\
44,4 \\
64,9\end{array}$} & \multirow{3}{*}{$\frac{\text { Valor de } p}{0,001^{*}}$} \\
\hline \multirow{2}{*}{\multicolumn{2}{|c|}{ Área do limite de estabilidade (LOS) }} & & & & & \\
\hline & & & & & & \\
\hline \multirow{20}{*}{ Área de elipse $\left(\mathrm{cm}^{2}\right)$} & SFOA & $\mathrm{F}$ & 1,93 & 1,79 & 0,78 & 0,554 \\
\hline & & M & 2,43 & 2,05 & 1,65 & \\
\hline & SFOF & $\mathrm{F}$ & 1,94 & 1,66 & 1,43 & 0,168 \\
\hline & & M & 2,65 & 2,08 & 2,13 & \\
\hline & SEOF & $\mathrm{F}$ & 8,83 & 7,60 & 6,72 & 0,554 \\
\hline & & M & 8,82 & 6,96 & 4,14 & \\
\hline & SFS & $\mathrm{F}$ & 1,47 & 1,14 & 1,09 & 0,187 \\
\hline & & M & 2,08 & 1,31 & 1,73 & \\
\hline & SFOD & $\mathrm{F}$ & 1,98 & 1,55 & 2,00 & 0,655 \\
\hline & & M & 2,20 & 1,62 & 1,67 & \\
\hline & SFOE & $\mathrm{F}$ & 1,42 & 0,98 & 1,04 & 0,236 \\
\hline & & M & 1,94 & 1,29 & 1,86 & \\
\hline & SFOB & $\mathrm{F}$ & 1,51 & 1,09 & 1,17 & $0,086^{\#}$ \\
\hline & & M & 2,05 & 1,59 & 1,36 & \\
\hline & SFOC & $\mathrm{F}$ & 1,69 & 1,33 & 1,40 & 0,109 \\
\hline & & M & 2,24 & 2,05 & 1,48 & \\
\hline & SFIVVDH & $\mathrm{F}$ & 2,25 & 2,05 & 1,23 & $0,007^{*}$ \\
\hline & & M & 4,66 & 2,82 & 4,54 & \\
\hline & SFIVVDV & $\mathrm{F}$ & 2,37 & 1,81 & 1,69 & $<0,001^{*}$ \\
\hline & & M & 5,49 & 4,51 & 3,74 & \\
\hline \multirow{20}{*}{ Velocidade de oscilação (cm/s) } & SFOA & $\mathrm{F}$ & 0,72 & 0,74 & 0,16 & 0,171 \\
\hline & & M & 0,77 & 0,62 & 0,60 & \\
\hline & SFOF & $\mathrm{F}$ & 0,88 & 0,87 & 0,26 & 0,362 \\
\hline & & M & 0,97 & 0,88 & 0,34 & \\
\hline & SEOF & $\mathrm{F}$ & 2,49 & 2,32 & 0,67 & 0,587 \\
\hline & & M & 2,32 & 2,25 & 0,43 & \\
\hline & SFS & $\mathrm{F}$ & 0,87 & 0,85 & 0,31 & 0,587 \\
\hline & & M & 0,90 & 0,85 & 0,27 & \\
\hline & SFOD & $\mathrm{F}$ & 0,78 & 0,76 & 0,24 & 0,357 \\
\hline & & M & 0,85 & 0,75 & 0,28 & \\
\hline & SFOE & $\mathrm{F}$ & 0,78 & 0,75 & 0,26 & 0,420 \\
\hline & & M & 0,86 & 0,75 & 0,30 & \\
\hline & SFOB & $\mathrm{F}$ & 0,81 & 0,82 & 0,22 & 0,662 \\
\hline & & M & 0,87 & 0,82 & 0,31 & \\
\hline & SFOC & $\mathrm{F}$ & 0,81 & 0,73 & 0,30 & 0,286 \\
\hline & & M & 0,93 & 0,87 & 0,38 & \\
\hline & SFIVVDH & $\mathrm{F}$ & 1,04 & 0,93 & 0,40 & $<0,001^{*}$ \\
\hline & & M & 1,45 & 1,29 & 0,57 & \\
\hline & SFIVVDV & $F$ & 1,21 & 1,15 & 0,46 & $<0,001^{*}$ \\
\hline & & $M$ & 1,83 & 1,73 & 0,53 & \\
\hline
\end{tabular}

*Valores significativos ( $\mathrm{p} \leq 0,05)$ - Teste de Mann-Whitney

\# Valores de $p$, que por estarem próximos do limite de aceitação, tendem a ser significativos

Legenda: $F$ = grupo feminino; $M$ = grupo masculino; SFOA = superfície firme de olhos abertos; SFOF = superfície firme de olhos fechados; SEOF = superfície espuma de olhos fechados; SFS = superfície firme movimento sacádico; SFOD = superfície firme barras optocinéticas na direção horizontal da esquerda à direita; SFOE = superfície firme barras optocinéticas na direção horizontal da direita à esquerda; SFOB = superfície firme barras optocinéticas na direção vertical de cima para baixo; SFOC = superfície firme barras optocinéticas na direção vertical de baixo para cima; SFIVVDH = superfície firme interação vestíbulo-visual na direção horizontal da esquerda à direita; SFIVVDV = superfície firme interação vestíbulo-visual na direção vertical de baixo para cima 
Os achados à posturografia deste estudo são de difícil comparação quantitativa com os de outros tipos de posturografia, uma vez que são utilizados diferentes parâmetros e procedimentos de avaliação. Para os estudos realizados ${ }^{(20,21)} \mathrm{com}$ o BRUTM foi utilizado um grupo controle para a comparação com os resultados obtidos nos grupos de pesquisas em pacientes com doença de Menière ${ }^{(21)} \mathrm{e} \mathrm{em} \mathrm{pacientes} \mathrm{com} \mathrm{esclerose} \mathrm{múltipla}{ }^{(20)}$, com médias de idade de 45,55 (DP=12,36 anos, variando entre 33 e 57 anos) e 34,91 ( $D P=13,97$ anos, variando entre 20 e 48 anos), respectivamente. Em contrapartida, o presente estudo apresentou média etária de 21,30 (DP=2,07 anos, variando entre 19 e 23 anos, de acordo com critério de inclusão), evidenciando diferença entre os estudos quanto à faixa etária ${ }^{(20,21)}$.

Ao comparar os valores dos estudos supracitados ${ }^{(20,21)}$ com o presente estudo, verificou-se que os valores médios do LOS obtidos no grupo controle do estudo com pacientes com doença de Menière - GCDM foram próximos aos do grupo controle do estudo de pacientes com esclerose múltipla - GCEM. Porém, são valores distantes dos obtidos neste presente estudo. Isto pode ser explicado pelo fato de que o grupo controle do presente estudo apresenta faixa etária inferior ao GCDM e ao GCEM.

Para a área de elipse, observou-se que o mesmo acontece para os seguintes estímulos sensoriais: superfície firme de olhos abertos, superfície firme de olhos fechados, com barras optocinético para a direita, com interação visuovestibular na direção horizontal e na direção vertical. Já para o estímulo superfície espuma de olhos fechados o GCEM é próximo ao do presente estudo, distanciando do valor médio do GCDM. Nos demais estímulos (superfície firme movimento sacádico, com barras optocinético para a esquerda, para baixo e para cima) os valores médios nos três estudos são próximos.

Quanto à velocidade de oscilação os valores médios obtidos neste estudo não foram muito distantes dos achados no $\operatorname{GCEM}^{(20)}$ e no GCDM $^{(21)}$.

Em nossa casuística, a posturografia do BRUTM mostrou que os valores da área do LOS (Tabela 2) do grupo feminino foram inferiores aos do grupo masculino. Esta diferença pode ser explicada pela estatura dos pacientes do gênero masculino que, em geral, foi superior à do grupo feminino, já que quanto mais alto o indivíduo, maior sua base de sustentação. Não foram encontradas citações da literatura sobre a área do LOS em pacientes adultos jovens à posturografia do equipamento BRUTM.

Estudo com a posturografia dinâmica do tipo "FoamLaser" (16) apresentou diferença no teste que mede as oscilações corporais - Teste de Organização Sensorial (TOS), em função do gênero e da faixa etária dos indivíduos, sendo que as mulheres apresentaram melhores resultados, nas diferentes faixas etárias. Tais achados e os resultados do presente estudo demonstram que há necessidade de se obter valores de normalidade diferenciados de acordo com o gênero.

A diferença de oscilação entre homens e mulheres pode ser explicada pela redução das respostas somatossensoriais, associadas à diminuição do grau de potência muscular. Isto é mais significativo em mulheres, uma vez que estas sofrem maiores perdas nas quantidades de massa magra e de força muscular do que os homens da mesma idade ${ }^{(30)}$. Além disso, a relação entre informação sensorial e ação motora é outro fator que pode influenciar no comportamento menos estável com o avançar da idade ${ }^{(22,30)}$.

Os valores da área de elipse, para a prova com estímulo sensorial em superfície firme com barras optocinéticas para baixo, apresentaram diferença em relação aos gêneros. Não foram encontradas citações da literatura sobre área de elipse em pacientes adultos jovens à posturografia do BRUTM.

Os valores da área de elipse e da velocidade de oscilação em duas condições avaliadas à posturografia do BRUTM no grupo feminino apresentaram diferença quando comparados aos do grupo masculino. Observou-se, também, que os pacientes do grupo feminino apresentaram pior desempenho nas provas com movimento de cabeça associado. Assim, a posturografia do BRUTM foi sensível para captar alterações do equilíbrio corporal em pacientes sem alterações evidentes à marcha. Não foram encontradas citações da literatura sobre alterações da Velocidade de Oscilação em pacientes adultos jovens à posturografia do BRUTM.

Nossos resultados indicaram que a posturografia com estímulos de realidade virtual é um método que provê informações relevantes sobre o equilíbrio corporal de adultos jovens hígidos. Os achados em relação aos valores da área do LOS, da área de elipse e da velocidade de oscilação na posturografia podem ser úteis não apenas em termos diagnósticos para a caracterização do distúrbio do equilíbrio corporal, como também para acompanhar a evolução da doença sob tratamento.

\section{CONCLUSÃO}

Os achados à posturografia com estímulos de realidade virtual em adultos jovens hígidos evidenciam que os parâmetros centro de pressão, limite de estabilidade e velocidade da oscilação apresentam diferenças entre os gêneros, e portanto, devem ser considerados separadamente.

\section{REFERÊNCIAS}

1. Ganança MM, Caovilla HH, Ganança FF, Doná F, Branco F, Paulino CA, et al. Como diagnosticar e tratar vertigem. Rev Bras Med. 2008;65(1):614.

2. Sataloff RT, Hawkshaw MJ, Mandel H, Zwislewski AB, Armour J, Mandel S. Abnormal computerized dynamic posturography findings in dizzy patients with normal ENG results. Ear Nose Throat J. 2005;84(4):212-4.

3. Terekhov Y. Stabilometry and some aspects of its applications--a review. Biomed Eng. 1976;11(1):12-5.

4. Evans MK, Krebs DE. Posturography does not test vestibulospinal function. Otolaryngol Head Neck Surg. 1999;120(2):164-73.

5. Longridge NS, Mallinson AI. "Across the board" posturography abnormalities in vestibular injury. Otol Neurotol. 2005;26(4):695-8.

6. Soto A, Labella T, Santos S, Río MD, Lirola A, Cabanas E, et al. The usefulness of computerized dynamic posturography for the study of equilibrium in patients with Meniere's disease: correlation with clinical and audiologic data. Hear Res. 2004;196(1-2):26-32.

7. Teszler CB, Ben-David J, Podoshin L, Sabo E. Sonovestibular symptoms evaluated by computed dynamic posturography. Int Tinnitus J. 2000;6(2):140-53.

8. Gianoli G, McWilliams S, Soileau J, Belafsky P. Posturographic performance in patients with the potential for secondary gain. Otolaryngol Head Neck Surg. 2000;122(1):11-8. 
9. Lipp M, Longridge NS. Computerised dynamic posturography: its place in the evaluation of patients with dizziness and imbalance. J Otolaryngol. 1994;23(3):177-83.

10. Medeiros IR, Bittar RS, Pedalini ME, Lorenzi MC, Kiil MA, Formigoni LG. Avaliação do tratamento dos distúrbios vestibulares na criança através da posturografia dinâmica computadorizada: resultados preliminares. J Pediatr (Rio J). 2003;79(4): 337-42.

11. BRU. Unidade de Reabilitação do Equilíbrio. Manual do usuário. Versão 1.0.7. Versão do Software: 1.3.5.0. Montevideo: Medicaa; 2006. 132p.

12. Di Girolamo S, Picciotti P, Sergi B, Di Nardo W, Paludetti G, Ottaviani F. Vestibulo-ocular reflex modification after virtual environment exposure. Acta Otolaryngol. 2001;121(2):211-5.

13. Rosa JL, Perracini MR, Ganança FF. Estabilometria em pacientes com doença de Ménière. Acta ORL. 2006;24(4):232-8.

14. Ojala M, Matikainen E, Juntunen J. Posturography and the dizzy patient: a neurological study of 133 patients. Acta Neurol Scand. 1989;80(2):11822.

15. Novalo ES, Pedalini ME, Bittar RS, Lorenzi MC, Bottino MA. Posturografia dinâmica computadorizada: avaliação quantitativa de pacientes com vestibulopatia tratados por meio de reabilitação vestibular. Arq Int Otorrinolaringol. 2008;12(2): 253-7.

16. Ruwer SL. Estudo da posturografia dinâmica "Foam-Laser" em indivíduos normais com idades entre 14 e 60 anos [dissertação]. Santa Maria(RS): Universidade Federal de Santa Maria; 2006.

17. Girardi M, Konrad HR, Amin M, Hughes LF. Predicting fall risks in an elderly population: computer dynamic posturography versus electronystagmography test results. Laryngoscope. 2001;111(9):1528-32.

18. Fujita T, Nakamura S, Ohue M, Fujii Y, Miyauchi A, Takagi Y, et al. Effect of age on body sway assessed by computerized posturography. J Bone Miner Metab. 2005;23(2):152-6.

19. Bittar RS. Como a posturografia dinâmica computadorizada pode nos ajudar nos casos de tontura? Arq Int Otorrinolaringol. 2007;11(3):330-3
20. Kessler N. Da posturografia na esclerose múltipla [dissertação]. São Paulo(SP): Universidade Federal de São Paulo; 2008.

21. Cusin FS. Posturografia do Balance Rehabilitation Unit $\left(\mathrm{BRU}^{\mathrm{TM}}\right)$ na doença de Menière [dissertação]. São Paulo(SP): Universidade Federal de São Paulo; 2009.

22. Freitas Júnior PB. Características comportamentais do controle postural de jovens, adultos e idosos [dissertação]. Rio Claro(SP): Universidade Estadual Paulista; 2003.

23. Montero EF, Zanchet DJ. Realidade virtual e a medicina. Acta Cir Bras. 2003;18(5):489-90.

24. Colombo G. Realidade virtual utilizada na reabilitação do AVC. Rev Saúde Med [Internet] Abr 2008; [citado 17 Nov 2008]. Disponível em: http://www.criativopunk.com.br/2008/04/05/realidade-virtual-utilizadana-reabilitacao-do-avc

25. Keshner EA. Virtual reality and physical rehabilitation: a new toy or a new research and rehabilitation tool? J Neuroeng Rehabil. 2004;1(1):8.

26. Suárez H, Suárez A, Lavinsky L. Postural adaptation in elderly patients with instability and risk of falling after balance training using a virtualreality system. Int Tinnitus J. 2006;12(1):41-4.

27. Gazzola JM, Doná F, Ganança MM, Suarez H, Ganança FF, Caovilla HH Realidade virtual na avaliação e reabilitação dos distúrbios vestibulares. Acta ORL. 2009;27(1):22-7.

28. Norré ME, Forrez G. Posture testing (posturography) in the diagnosis of peripheral vestibular pathology. Arch Otorhinolaryngol. 1986;243(3):186-9.

29. Tossavainen T, Toppila E, Pyykkö I, Forsman PM, Juhola M, Starck J. Virtual reality in posturography. IEEE Trans Inf Technol Biomed. 2006;10(2):282-92.

30. Almeida AP, Veras RP, Doimo LA. Avaliação do equilíbrio estático e dinâmico de idosas praticantes de hidroginástica e ginástica. Rev Bras Cineantropom Desempenho Hum. 2010;12(1):55-61. 